\title{
Acoustophoretic cell and particle trapping on microfluidic sharp edges
}

\author{
Ivo Leibacher ${ }^{1} \cdot$ Philipp Hahn $^{1} \cdot$ Jürg Dual $^{1}$
}

Received: 28 May 2015 / Accepted: 24 July 2015 / Published online: 14 August 2015

(C) Springer-Verlag Berlin Heidelberg 2015

\begin{abstract}
Cell and particle trapping experience a rising interest in a microfluidic context. Trapping of cells in suspension at distinct locations in a microfluidic domain is relevant for cell biological lab-on-a-chip systems, where a trap provides a well-definable microenvironment for cell response studies. This paper reports a novel acoustophoretic cell trapping effect on oscillating sharp edge structures which protrude into a microfluidic channel. These edge structures (125-250 $\mu \mathrm{m}$ length, $10-80 \mu \mathrm{m}$ width in the experiments) were found to attract cells and particles strongly and reliably upon simple piezoelectric excitation around $1 \mathrm{MHz}$. The method is contact- and label-free, robust, and biocompatible. The physical trapping effect is experimentally characterized, and a numerical model is proposed, based on the theory of acoustic radiation forces.
\end{abstract}

Keywords Acoustofluidics · Acoustophoresis · Acoustic radiation force $\cdot$ Acoustic streaming $\cdot$ Cell trapping

\section{Introduction}

The advent of microfluidic tools allows to access cells and particles from a new perspective. In recent

Electronic supplementary material The online version of this article (doi:10.1007/s10404-015-1621-1) contains supplementary material, which is available to authorized users.

Ivo Leibacher

leibacher@imes.mavt.ethz.ch

1 Department of Mechanical and Process Engineering, Institute of Mechanical Systems (IMES), Swiss Federal Institute of Technology (ETH Zurich), Tannenstrasse 3, 8092 Zurich, Switzerland years, chip-based cell biology research has been closely accompanied by the development of microfluidic devices for cell processing (Haeberle et al. 2012; Salieb-Beugelaar et al. 2010; West et al. 2008; Whitesides 2006), where the physics at the microscale has been leveraged for functionality regarding cell, particle, and fluid handling.

More specific, microfluidic particle trapping experiences an increasing interest in the lab-on-a-chip community. The trapping of cells and particles (Johann 2006; Nilsson et al. 2009) out of a suspension onto distinct locations is an important unit operation for cell capturing, cultivation, and analysis in diagnostics and research. Trapping provides well-defined chemical thermal microenvironments for experimental cell response studies, cell-cell interactions, cell enrichment, biosensors, perfused 3D cell culturing, exchange of cell's suspending medium, cell washing, and screening of the cells (Christakou et al. 2013; Evander et al. 2007; Li et al. 2014). The spatial control of the cells is pivotal in these applications.

Existing methods for microfluidic cell and particle trapping (Johann 2006; Nilsson et al. 2009) are dielectrophoretic trapping (DEP) (Voldman 2006), hydrodynamic trapping (Karimi et al. 2013), and optical tweezers (Grier 2003), furthermore magnetic trapping, and patch clamping. Compared to these methods, acoustic methods (Christakou et al. 2013; Evander et al. 2007; Evander and Nilsson 2012; Gralinski et al. 2014; Hammarström et al. 2010; Li et al. 2014; Manneberg et al. 2008; Vanherberghen et al. 2010) show several benefits: They can easily be integrated on chip level (unlike optical tweezers), they are simple to fabricate (no structured in-chip electrodes needed as for DEP), and they work on most cell and particle types (no dependency on magnetic/dielectric particle properties, acoustic properties are typically suitable). Furthermore they are 
contact-less, label-free, and biocompatible also in longterm studies (Vanherberghen et al. 2010).

In the paper at hand, a novel acoustic cell trapping mechanism is proposed, based on acoustic radiation forces. The method allows to attract cells (suspended in water) to oscillating sharp edge structures which protrude in a microfluidic channel (Leibacher and Dual 2014a, b). Besides the common characteristics of acoustic methods, this method has specific advantages: It does not need a reflector-bounded cavity to form a standing wave field as in the common design (Christakou et al. 2013; Evander et al. 2007; Evander and Nilsson 2012; Gralinski et al. 2014; Hammarström et al. 2010; Li et al. 2014; Manneberg et al. 2008; Vanherberghen et al. 2010), it allows to trap cells on an arbitrary position in a microfluidic channel, and it does not require a precise tuning of a fluid resonance frequency. Hence, the proposed method allows stable and robust trapping of cells and particles.

In literature, particle attraction to oscillating structures has previously been observed in centimeter-scaled setups: The attraction of $\sim 1 \mathrm{~mm}$ particles to oscillating sharp edges (Hu et al. 2004), rods (Liu and Hu 2009), and needles (Hu et al. 2007) was reported in the $\mathrm{kHz}$ range. In these cases, the described physical effects were also based on acoustic radiation forces, yet with a different structural setup and physical modeling. Compared to this previous work, the paper at hand aims at the microfluidic exploitation of similar effects for cell handling.

Besides particle trapping, oscillating sharp edges have further been reported for fluid mixing (Huang et al. 2013; Oberti et al. 2009) and pumping (Huang et al. 2014) by acoustic streaming. Due to the wealth of acoustofluidic phenomena around oscillating sharp edges, they hold promise to become a powerful actuation mechanism for various microfluidic tasks, similar to the success of oscillating bubbles (Hashmi et al. 2012; Marmottant and Hilgenfeldt 2004) as a microfluidic driving mechanism.

\section{Methods and materials}

The experiments were conducted in a microfluidic chip as illustrated in Fig. 1. The chip consisted of a $30 \mu \mathrm{m}$ deep microfluidic channel, where 24 sharp edges were protruding in the fluid domain. The sharp edges had a length of 125 or $250 \mu \mathrm{m}$ and a varying width of $10-80 \mu \mathrm{m}$. The silicon chip was covered by a glass plate, and it was filled manually by placing fluid drops with suspended cells on one of the two fluid ports. In $z$-direction, the edges ranged from the channel bottom up to the glass lid.

The ultrasound transducer consisted of a piezoelectric element, mounted underneath the device. Upon a harmonic electrical excitation, the transducer vibrated and thereby (a)

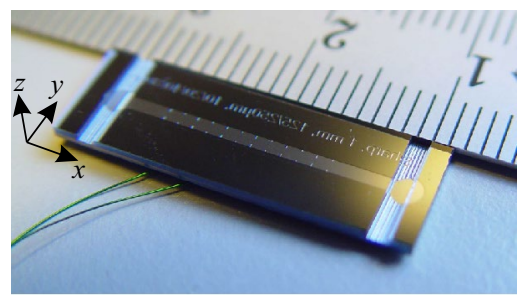

(b) Front side Fluidic channel, $x \times y \times z=$

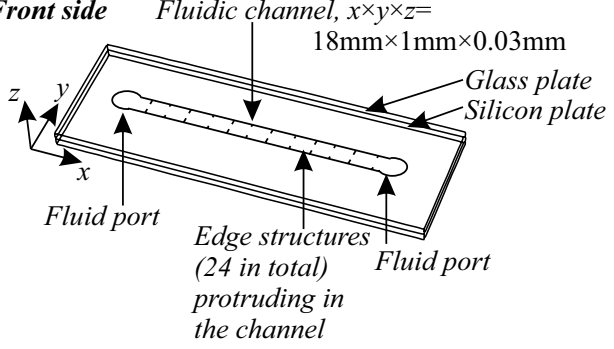

(c) Back side

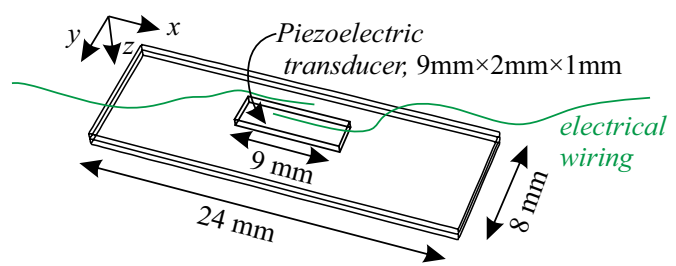

Fig. 1 a Photograph of the microfluidic silicon device front side with centimeter scales; $\mathbf{b}$ and $\mathbf{c}$ sketches of the microfluidic device. Sharp edges are protruding in the fluidic channel of $30 \mu \mathrm{m}$ depth

excited the whole device. The piezoelectric transducer was polarized in the $z$-direction, yet it induced vibrations in all three directions due to the direct and transverse piezoelectric effect, as experienced in transversal acoustofluidic devices (Lenshof et al. 2012). This vibration of the sharp edge caused the acoustofluidic effects which will be presented in the results chapter. In detail, the materials and methods are specified in the following:

Excitation: To excite a mechanical vibration, a piezoelectric transducer was cut to a size of $9 \mathrm{~mm} \times 2 \mathrm{~mm}$ out of a 1-mm-thick plate of Ferroperm piezoceramics (Ferroperm piezoceramics a/s) Pz26. This piezoelectric block was glued on the bottom of the device with conductive epoxy (Epo-Tek H20E). The glue layer provided a plane electrode on the top side of the piezoelectric block. Parallel to this electrode, a second plane electrode was provided by a thin conductive layer on the bottom side of the block (with normal in z-direction).

For electrical excitation, the top and bottom planar electrodes of the piezoelectric block were connected to a function generator (Tektronix AFG 3022B) with a power amplifier (ENI, 2100L) in between. The applied excitation voltages were in the range of $\sim 5-15 \mathrm{~V}_{\text {rms. }}$. 
Table 1 Modeled material properties

\begin{tabular}{ll}
\hline Copolymer particles (Oberti et al. 2007) & (Duke scientific) \\
Density & $\rho_{\mathrm{co}}=1050 \mathrm{~kg} / \mathrm{m}^{3}$ \\
Speed of sound & $c_{\mathrm{co}}=3000 \mathrm{~m} / \mathrm{s}$ \\
Diameter & $2 r_{\mathrm{co}}=11 \mu \mathrm{m}$ \\
$f_{1}, f_{2}$ & $0.76,0.034$ \\
Hollow glass particles (Leibacher et al. 2014) & $($ Kisker PBGH-18) \\
Averaged density & $\bar{\rho}_{\mathrm{ho}}=600 \mathrm{~kg} / \mathrm{m}^{3}$ \\
Diameter & $2 r_{\mathrm{ho}}=13.9 \mu \mathrm{m}$ \\
$f_{1}, f_{2}$ & $0.602,-0.362$ \\
Glass particles (Bruus 2012) & $($ Pyrex, simulated) \\
Density & $\rho_{\mathrm{py}}=2230 \mathrm{~kg} / \mathrm{m}^{3}$ \\
Young's modulus & $E_{\mathrm{py}}=63 \mathrm{GPa}$ \\
Poisson's ratio & $\nu_{\mathrm{py}}=0.22$ \\
Diameter & $2 r_{\mathrm{py}}=10 \mu \mathrm{m}$ \\
$f_{1}, f_{2}$ & $0.94,0.45$ \\
Water (Bruus 2012) & $\left(\right.$ at $\left.25^{\circ} \mathrm{C}\right)$ \\
Density & $\rho_{0}=998 \mathrm{~kg} / \mathrm{m}^{3}$ \\
Speed of sound & $c_{0}=1497 \mathrm{~m} / \mathrm{s}$ \\
Silicon & \\
Density (Bruus 2012) & $\rho_{\mathrm{si}}=2331 \mathrm{~kg} / \mathrm{m}^{3}$ \\
Speed of sound, [110] direction (McSkimin and & $c_{\mathrm{si}}=9133 \mathrm{~m} / \mathrm{s}$ \\
Andreatch 1964) & \\
\hline
\end{tabular}

Microfabrication: The microdevices based on a $24 \mathrm{~mm}$ $\times 8 \mathrm{~mm}$ silicon plate (wafer thickness $425 \mu \mathrm{m}$ ). A 1-mmwide fluidic channel was dry etched $30 \mu \mathrm{m}$ deep by an inductively coupled plasma system, sparing the edge geometries by a patterned photoresist. A glass plate (thickness $500 \mu \mathrm{m}$ ) was bonded anodically on top of the silicon. As confirmed by visual inspection, this glass plate also bonded to the top surface of the sharp edges, so they were clamped on the bottom as well as the top. The fluidic inlet and outlet were provided by several parallel cuts through the glass lid with a wafer saw.

Cells and particles: Yeast cell suspensions were prepared by diluting baker's yeast in DI water. The material properties of particles, the suspending water, and silicon are listed in Table 1 as modeled in the following numerical simulations.

Image acquisition: Videos and images were recorded with a high-speed camera (HiSpec 1 Mono, Fastec Imaging). The silicon devices were illuminated from above as in bright-field microscopy: The light of a LED lamp was introduced parallel to the optical axis by a half-transparent mirror. Because of the high reflectivity of silicon, this lighting setup resulted in bright illumination as required for high-speed imaging. For video analysis such as feature tracking (see later in Figs. 2d, 4d) as well as for the particle image velocimetry (PIV, in Fig. 6b), the software Xcitex ProAnalyst was employed.

\section{Results and discussion}

\subsection{Experimental results}

\subsubsection{Trapping and repulsion of particles by acoustic radiation forces}

The subject of this paper is the attraction and trapping of yeast cells on a microfluidic sharp edge, as it is shown in Fig. 2a-c. The image series shows the quick buildup of several hundreds of yeast cells on the tip of a silicon sharp edge, which protrudes in a microfluidic channel filled with suspending water. The driving force was an ultrasound transducer that was turned on at the start $t=0$ of the image series. The trapping occurred at an actuation frequency of $924 \mathrm{kHz}$ with $15 \mathrm{~V}_{\text {rms }}$ in this image series, yet the effect was observed at several other frequencies too, mostly in the range of $850-1000 \mathrm{kHz}$ in our setup as it will be discussed in Sect. 3.1.2.

Before a physical explanation of the effect is proposed in the following Sect. 3.2, more experimental results are presented to study the underlying physical effects. Figure $2 \mathrm{~d}$ shows an experiment at $897 \mathrm{kHz}$ excitation with $12 \mathrm{~V}_{\mathrm{rms}}$, where copolymer particles of $11 \mu \mathrm{m}$ size were used instead of yeast cells. Copolymer particles behave qualitatively like the cells and get attracted to the edge structure. Several seconds after the start of the ultrasonic excitation, the shown steady state occurred with stably trapped particles on the tip of the edge. The shadowy band around the edges and at walls resulted as the channel bottom is not completely even in this region, so the illumination light is not reflected into the camera.

With a video analysis, a dozen particle paths were tracked to visualize the kinematics of the particles by the red lines in the image. These particle paths are a fingerprint of the underlying force field, which is modeled numerically in Sect. 3.2. Particle speeds of $100-1000 \mu \mathrm{m} / \mathrm{s}$ were measured by image analysis. The particle speed results from a balance of forces between the Stokes' drag and the acoustic forces (Barnkob et al. 2010), which can thereby be calculated as 1e-11-1e-10 $\mathrm{N}$ in the experiment of Fig. 2d. This is a typical value compared to acoustophoresis with ultrasonic standing waves (Barnkob et al. 2010). However, the forces were increasing strongly in close proximity to the tip of the edge, as it can also be seen in the supplementary video where particles are accelerating quickly toward this equilibrium position. In contrary, the acoustic radiation forces in common ultrasonic standing wave traps are decreasing to zero toward the equilibrium position in a pressure node. Therefore, once a particle is trapped on a sharp edge, it is held back much stronger than in ultrasonic standing wave traps. 


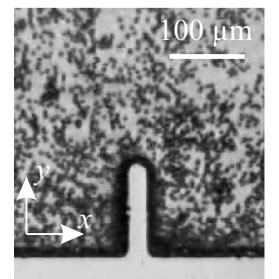

(a) $t=0 \mathrm{~s}$

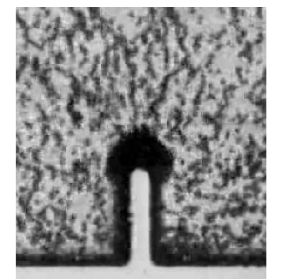

(b) $t=0.72 \mathrm{~s}$

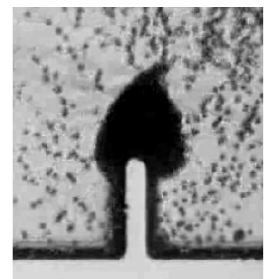

(c) $t=5.8 \mathrm{~s}$

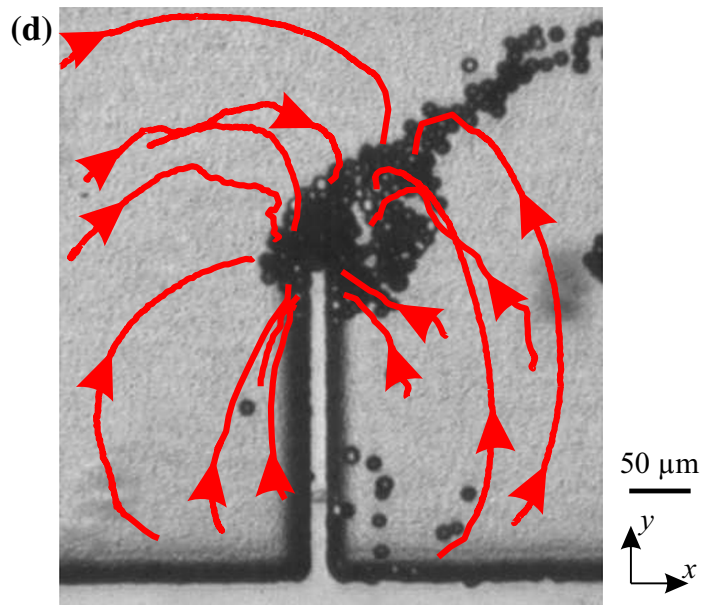

Fig. 2 a-c Time series of yeast cell trapping. Yeast cells (seen as dark dots) were suspended in a microfluidic channel. Upon mechanical excitation of $924 \mathrm{kHz}$ at time $t>0$, the yeast cells were attracted to the sharp edge of size $y \times x=125 \mu \mathrm{m} \times 20 \mu \mathrm{m}$ (Video 1 available online in the supporting electronic documents). d Trapping of $11 \mu \mathrm{m}$ copolymer particles on an edge structure of $250 \mu \mathrm{m} \times 10 \mu \mathrm{m}$ at $897 \mathrm{kHz}$. Red lines mark the tracked particle paths which correspond to the simulated streamlines in Fig. 8a (color figure online)
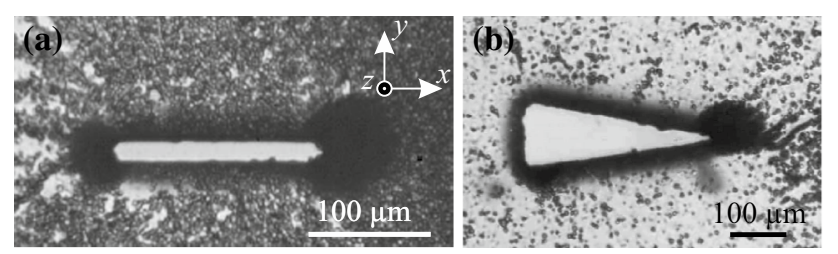

Fig. 3 a Yeast cell trapping on both sides of a sharp edge (size $125 \mu \mathrm{m} \times 10 \mu \mathrm{m}) 0.6 \mathrm{~s}$ after ultrasound was turned on (Video 1 available online in the supporting electronic documents). b On a triangle (325 $\mu \mathrm{m}$ height in $x$-direction, $100 \mu \mathrm{m}$ width in $y$-direction), cell trapping occurred only at the sharp angle of the right corner

In Fig. 3, two more sharp edge designs were placed freestanding in a channel of $16 \mathrm{~mm} \times 3 \mathrm{~mm} \times 30 \mu \mathrm{m}$. Here, best trapping was found at $676 \mathrm{kHz}$ in (a) and $678 \mathrm{kHz}$ in (b). These frequencies are lower than before due to a larger transducer size of $10 \mathrm{~mm} \times 3 \mathrm{~mm}$ with different transducer resonance frequency, as outlined in Sect. 3.1.2. Notably, for the triangle shape in Fig. 3b, trapping was only observed at the corner on the right side with a small angle.

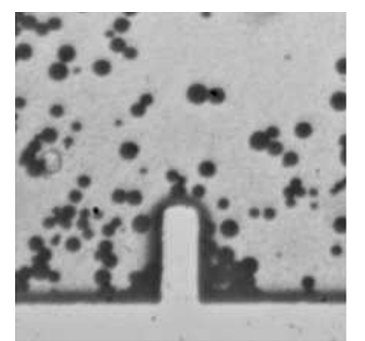

(a) $t=0 \mathrm{~s}$

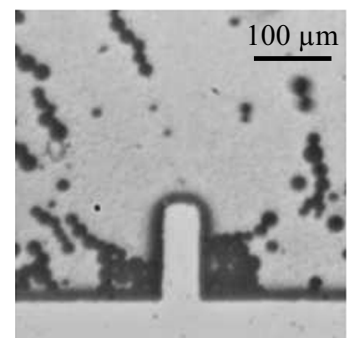

(c) $t=0.17 \mathrm{~s}$

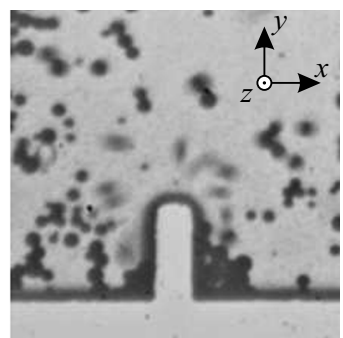

(b) $t=0.14 \mathrm{~s}$

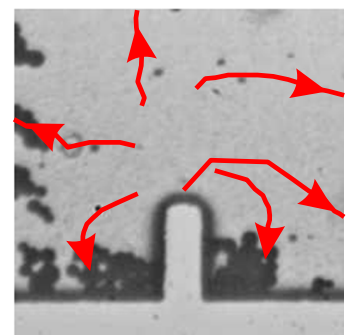

(d) $t=0.40 \mathrm{~s}$

Fig. 4 Time series of the repulsion of hollow particles from an oscillating edge $(125 \mu \mathrm{m} \times 40 \mu \mathrm{m})$ at $890 \mathrm{kHz}$. The $14 \mu \mathrm{m}$ particles were lighter than the suspending water. Red lines mark the tracked particle path (Video 2 available online in the supporting electronic documents).

After these similar observations on yeast cells and copolymer particles, Fig. 4 reports the behavior of hollow glass particles in the same experiment. From an acoustic viewpoint, their main difference to the yeast cells and copolymer particles is their low density which is even lower than the density of water (see Table 1). Contrary to copolymer and yeast, these hollow particles were not attracted but repelled from the oscillating sharp edge.

For the physical explanation of this phenomenon, several acoustofluidic effects were considered. From the video analysis, it is clear that the vibration of the chip structure imposed a force field on the particles. The force field acted selectively on the particles. It is plausible that the observed trapping forces were not caused by hydrodynamic drag of a flowing fluid. This follows because a force field on the fluid would result in closed streamlines, and it would act the same way on the hollow as on the full particles. Therefore, we believe the force field on the particles was caused by acoustic radiation forces as in "acoustophoresis." The model in Sect. 3.2 bases on this insight.

Regarding the viability of cells, acoustophoresis is known to be a gentle method. Viability-related effects such as cavitation, thermal stress, and acoustic streaming are well studied and have been discussed in a recent review (Wiklund 2012). Several research groups reported on acoustophoretic cell handling with no adverse effects on their viability, whereas the cells were 
exposed up to $72 \mathrm{~h}$ of ultrasound in microfluidic devices (Evander and Nilsson 2012; Vanherberghen et al. 2010). Nevertheless, viability considerations are justified, since ultrasound can harm cells, depending on the amplitude and frequency. In summary, this trapping mechanism can supposably be realized with amplitudes that are sufficiently low and frequencies that are sufficiently high not to damage cells.

\subsubsection{Vibrometry measurements}

To investigate the occurence of the trapping, further experimental characterization of the acoustofluidic device was considered. Figure 5a shows the frequency spectrum of a vibration analysis by laser vibrometry (Polytec OFV 505 and OFV 3001), Fig. 5b is an impedance measurement of the piezoelectric transducer (SinePhase Impedance Analyzer 16777k). These experimental characterization methods have been described in detail in our former work (Dual et al. 2012).

For the measurement of the transfer function between applied voltage on the transducer and device vibration in Fig. 5a, five randomly distributed points on the surface of the silicon device were measured with a laser vibrometer. A frequency sweep on the transducer voltage led to an excitation of all frequencies in the plotted range. The angle between the device and the incident laser was adjusted for a perfect back reflection on the silicon surface. This led to an excellent estimate of the coherence function, $\hat{\gamma}^{2}(f) \sim 1$ [detailed in Dual et al. (2012)] for the shown transfer function.

The plot denotes the highest mechanical vibration amplitudes in the range of $850-1000 \mathrm{kHz}$ due to electro-mechanical resonances of the system in this frequency range. This assumption is supported by the impedance measurement in Fig. 5 b, where the positive and negative peaks also denote an electro-mechanical resonance in this range, as described in literature (Dual et al. 2012).

The frequency range of $850-1000 \mathrm{kHz}$ with maximal measured vibration coincides with the range where the highest particle attraction/repulsion on the edges was observed in the experiments of the last section. This means there is a link between maximal device/sharp edge vibration and the acoustofluidic trapping/repulsion effect. Consequently, the trapping depends mainly on an electromechanical resonance of the transducer and the attached structure. This is a difference to the common acoustophoretic trap design (Evander and Nilsson 2012) where the particle manipulation is primarily based on a resonance of the fluidic domain with a sharp bandwidth. In these devices, an ultrasonic standing wave is formed between sound-reflecting channel or cavity walls (Lenshof et al. 2012) which causes cells to align on pressure nodal lines along the channel, whereby the resonance frequency has to be matched to the fluid channel width and fluid properties. Differently, the trapping on the sharp edge is not bound to such fluidic resonance conditions, as it will also be discussed on the numerical model in Sect. 3.2.

To explain the trapping effect, also structural resonances of the edge structure itself have to be considered; however, these eigenmodes are expected to occur at higher frequencies than applied here. Furthermore, temperature-dependent effects
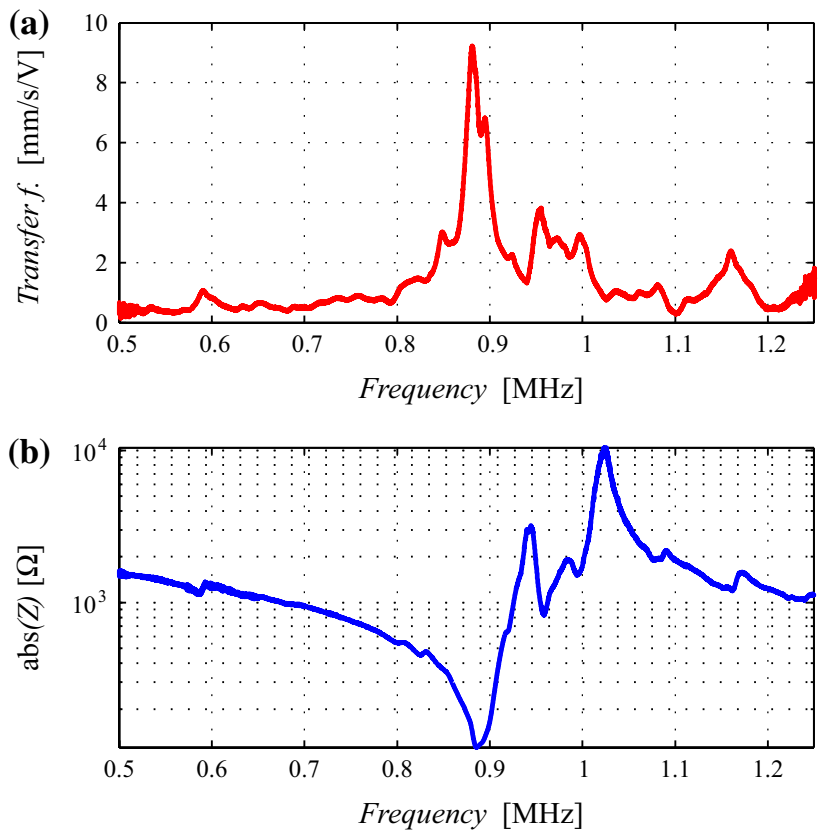

Fig. 5 a Transfer function between applied voltage on the piezoelectric element and the device velocity, averaged on five points on the device surface. At the electro-mechanical resonance of $890 \mathrm{kHz}$, best trapping performance was observed in this device. $\mathbf{b}$ Corresponding measurement of the electrical impedance $Z$ of the piezoelectric transducer

(a)

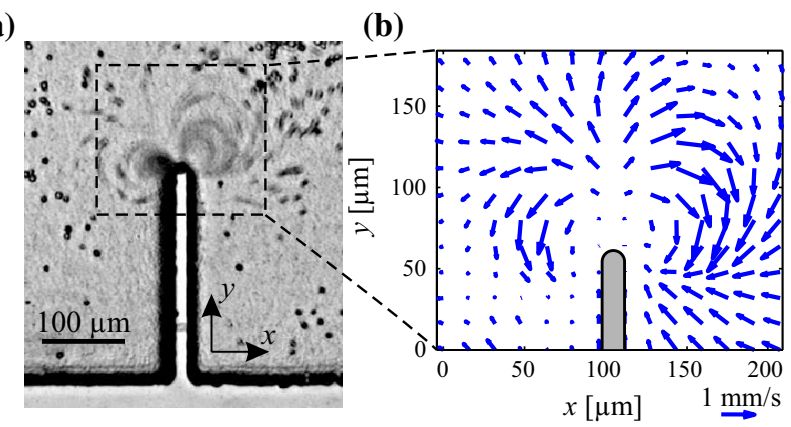

Fig. 6 a Acoustic streaming at an edge of $250 \mu \mathrm{m} \times 10 \mu \mathrm{m}$. The yeast cells are moved by two counter-rotating vortices of the fluid at the tip of the sharp edge (Video 3 available online in the supporting electronic documents). b Velocity field of the acoustic streaming around the edge structure in a. The plot was calculated by particle image velocimetry (PIV) 
(Augustsson et al. 2011) might shift the resonance frequency for some kilohertz during some experiments, since the piezoelectric transducer acts as a heat source on the device.

\subsubsection{Acoustic streaming}

Besides trapping and repulsion of particles by radiation forces, a further phenomenon was observed on some of the 24 sharp edges on a chip. Figure 6a shows yeast cells that are rotating fast in two counter-rotating vortices at the tip of the edge at $15 \mathrm{~V}_{\text {rms }}$ excitation. In Fig. $6 \mathrm{~b}$, a particle image velocimetry over 1636 images, recorded over a time span of $4.1 \mathrm{~s}$, further outlines the observed velocity field.

Comparing this effect with the work in literature (Huang et al. 2013; Lieu et al. 2012), this phenomenon can be identified as acoustic streaming. Acoustic streaming is a steady fluid flow driven by acoustic oscillations (Lighthill 1978; Sadhal 2012). The nonlinear, time-averaged effect can be categorized in boundary-layer-driven and bulk-attenuation-driven streaming. Boundary-layer-driven streaming is localized in areas of acoustic absorption in the viscous boundary layer, e.g., at vibrating walls, bubbles, or sharp edges. Acoustic streaming has typically closed streamlines of the fluid flow and is visible as a vortex or couples of counter-rotating vortices. Drag forces (Stokes' drag) cause suspended particles in the fluid to follow the streamlines. The acoustic streaming is not a subject of this paper; however, it is mentioned here for completeness and in order to distinguish it from the cell trapping mechanism.

Similar acoustic streaming around sharp edges has been reported for microfluidic mixing (Huang et al. 2013) and pumping (Huang et al. 2014), and also particle trapping (Lieu et al. 2012) (named "hydrodynamic tweezers"). Acoustic streaming can also occur as cavitation microstreaming around stably oscillating microbubbles (Wiklund et al. 2012) and around air bubbles in general (Ahmed et al. 2009; Marin et al. 2015; Sadhal 2012; Wiklund et al. 2012), which might also be considered as a cause of observed streaming. However, we did not observe bubbles in our experiments, which would cause the streaming to be less stable. Numerical models of acoustic streaming will be discussed in Sect. 3.2.

On the sharp edges of our microfluidic device, we experienced mostly the trapping/repulsion behavior by acoustic radiation forces. The acoustic streaming occurred less frequently but predominantly with more dilute suspensions on sharper, thin edge structures with small particles. Sometimes both radiation and streaming effects were visible, as in the first part of the supplementary Video 1. However, currently we cannot claim to be able to predict or control whether the radiation force or the streaming dominates the particle dynamics on a specific sharp edge in our devices. Presumably it depends on the position of a sharp edge with respect to the transducer and on the exact geometry of the edge. With the shown excitation by a relatively large piezoelectric block, acoustofluidic devices are known to have quite a nonuniform displacement field over a channel (Dual and Möller 2012). This might influence the complex acoustic streaming behavior, since it depends on the vibration direction of the sharp edge (Nama et al. 2014). Further, the balance between streaming and radiation forces depends on the particle size, the contrast factor, the frequency, and the fluid properties (Rogers and Neild 2011). Future work might address a more defined excitation method to study the competition between radiation and streaming forces, as also discussed in the next section.

\subsection{Numerical modeling}

Based on the insights from the last chapter, here a model is discussed for the acoustic radiation force around an oscillating sharp edge.

Before the model of the sharp edge is discussed, the theory of acoustic radiation forces is recapitulated in short. The acoustic radiation force on particles $\mathbf{F}=-\nabla U$ can be approximated by the gradient of the Gor'kov potential $U$. The Gor'kov potential (Gor'kov 1962) is valid for particles with a radius much smaller than the acoustic wavelength, $r \ll \lambda$. In the acoustic domain, the potential $U$ reads (Bruus 2012; Gor'kov 1962):

$U=2 \pi r^{3} \rho_{0}\left(\frac{\left\langle p_{1}^{2}\right\rangle}{3 \rho_{0}^{2} c_{0}^{2}} f_{1}-\frac{\left\langle v_{1}^{2}\right\rangle}{2} f_{2}\right)$

with the density $\rho_{0}$ and the speed of sound $c_{0}$ of the fluid (here water), the first-order pressure field $p_{1}(x, y, z)$, the first-order velocity (magnitude) field $v_{1}(x, y, z)$ and the material-dependent factors $f_{1}, f_{2}$. Time averaging is denoted by $\langle$.$\rangle .$

The first factor $f_{1}$ is given by

$f_{1}=1-\frac{\kappa_{p}}{\kappa_{0}}$

with the compressibility $\kappa$ and the indices $p$ of the particle material and 0 of the surrounding water. The compressibilities of the suspending fluid and a fluidic or solid particle are calculated as

$\kappa_{0}=\frac{1}{\rho_{0} c_{0}^{2}}, \quad \kappa_{p}=\frac{1}{K}=\frac{3(1-2 v)}{E}$

with the bulk modulus $K$, Young's modulus $E$, and Poisson's ratio $v$ of the elastic solid.

The second factor $f_{2}$ determines the influence of the velocity field in the Gor'kov potential. It is given as

$f_{2}=\frac{2\left(\rho_{p}-\rho_{0}\right)}{2 \rho_{p}+\rho_{0}}$ 
(a)
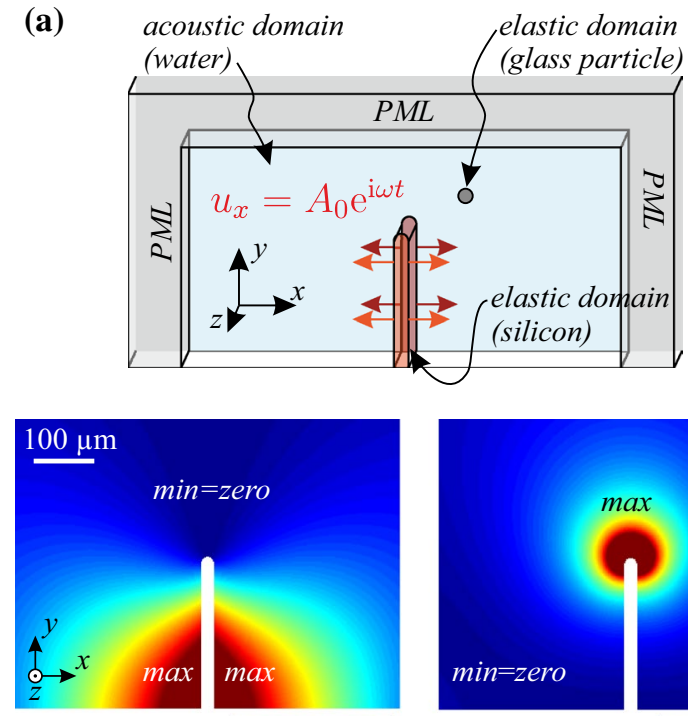

(b) $<p_{1}^{2}>(x, y)$

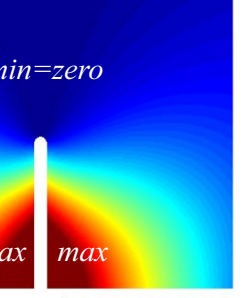

0

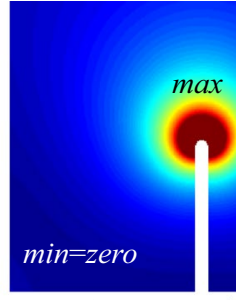

(c) $<v_{1}^{2}>(x, y)$

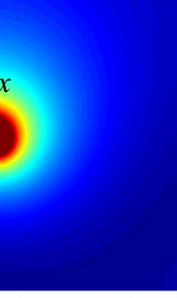

$0 \quad \max$

Fig. 7 a Sketch of the simulated 3D model with a harmonically oscillating edge in a fluid domain surrounded by perfectly matched layers (PML) and hard-wall boundary conditions at the top and bottom. Results of the simulated acoustic fields around the vibrating edge $(250 \mu \mathrm{m} \times 20 \mu \mathrm{m}, 900 \mathrm{kHz})$ are in $\mathbf{b}$ the time-averaged squared pressure $\left\langle p_{1}^{2}\right\rangle$ and in $\mathbf{c}$ the time-averaged squared velocity $\left\langle v_{1}^{2}\right\rangle$

According to these equations, particles in the acoustic domain are attracted to the minimum of the Gor'kov potential $U$. Generally speaking, as outlined in earlier work (Leibacher et al. 2014), a positive/negative factor $f_{1}$ contributes forces toward the minima/maxima of the $\left\langle p_{1}^{2}\right\rangle$ field (pressure nodes/antinodes), respectively. A positive/negative factor $f_{2}$ contributes forces toward the maxima/minima of the $\left\langle v_{1}^{2}\right\rangle$ field (velocity antinodes/nodes), respectively.

A common value to characterize the particle behavior is the acoustophoretic contrast factor (Yosioka and Kawasima 1955) $\Phi=\frac{f_{1}}{3}+\frac{f_{2}}{2}$. However, $\Phi$ is mostly significant for the fields of one-dimensional ultrasonic standing waves. Therefore, in our case of two-dimensional fields, the factors $f_{1}$ and $f_{2}$ rather than $\Phi$ are relevant.

To model the force field around the edge structures, a 3D simulation was built with the numerical software Comsol Multiphysics. Figure 7 a illustrates the model which consisted of a linear acoustic domain (fluid, sketched blue) and a linear elastic domain modeling the silicon sharp edge. In the time-harmonic simulation, the oscillation of the edge structure was imposed with an acceleration boundary condition on the top and bottom surface of the edge (in the $x y$ plane, sketched red in Fig. $7 \mathrm{a}$, with harmonic displacement field $u_{x}$ and amplitude $A_{0}$ ). This modeling was chosen since the top surface of the silicon edge was anodically bonded to the glass lid. Even though the sharp edge is believed to vibrate in all directions

experimentally, here it was modeled to vibrate only in $x$-direction, since additional vibration in $y$ - and $z$-direction was not significantly changing the fields and effects in this simulation.

Water-silicon and water-glass interfaces at the channel top and bottom surfaces (in the $x y$ plane) were modeled as hard-wall conditions (Bruus 2012; Leibacher et al. 2014) due to their high difference in characteristic acoustic impedance. To represent an infinite fluidic domain, it was surrounded by perfectly matched layers (PML) which absorb the majority of the outgoing waves. The depth in $z$-direction was set to $30 \mu \mathrm{m}$ as in the experiments.

The chosen nonviscous model is clearly a simplification, for now neglecting acoustic streaming effects. The chosen vibration boundary condition is also only a first approximation, neglecting the influence of the wave propagation problem from the transducer to the channel. However, the simple model can describe principal working mechanisms of the cell trapping phenomenon.

Figure $7 \mathrm{~b}$ reports a simulation result at an excitation frequency of $f=\omega /(2 \pi)=900 \mathrm{kHz}$. The time-averaged squared first-order pressure field $\left\langle p_{1}^{2}\right\rangle(x, y)$ is plotted, as it appears in the Gor'kov potential, Eq. 1. The maxima in this plot lie on the left and right side of the edge. These maxima are comprehensible from the chosen excitation $u_{x}$, which leads to harmonically alternating maxima/minima of the instantaneous pressure field $p_{1}$ on the left and right side of the sharp edge. At the outermost point of the edge, $p_{1}$ and accordingly $\left\langle p_{1}^{2}\right\rangle$ remain zero.

In linear acoustics with phasor notation, the velocity field in Fig. 7c follows from a gradient of the pressure field (Bruus 2012), as also implemented in Comsol Multiphysics.

The time-averaged squared first-order velocity field $\left\langle v_{1}^{2}\right\rangle(x, y)$ has its maximum at the tip of the sharp edge, since the movement $u_{x}$ of the sharp edge causes the fluid to move around this tip from the left side to the right side of the sharp edge and vice versa.

To reduce singularity issues, the tip of the sharp edge was rounded (with radius $10 \mu \mathrm{m}$ ) in our model, which results in numerically and probably also experimentally different results compared to a distinct corner with corner radius $\rightarrow 0$.

The fields in Fig. 7b, c lead to an explanation of the yeast cell attraction to the edge: As most common particles (e.g., copolymer, glass, polystyrene), most cells such as yeast exhibit $f_{1}>0, f_{2}>0$ because they are less compressible and heavier than the suspending water. Therefore, these cells are attracted to a minimum of $\left\langle p_{1}^{2}\right\rangle$ and a maximum of $\left\langle v_{1}^{2}\right\rangle$, as described by Gorkov's equation, Eq. 1 . This pressure minimum and the velocity maximum are both located on the outermost point of the edge, which explains the yeast cell attraction to this point. 


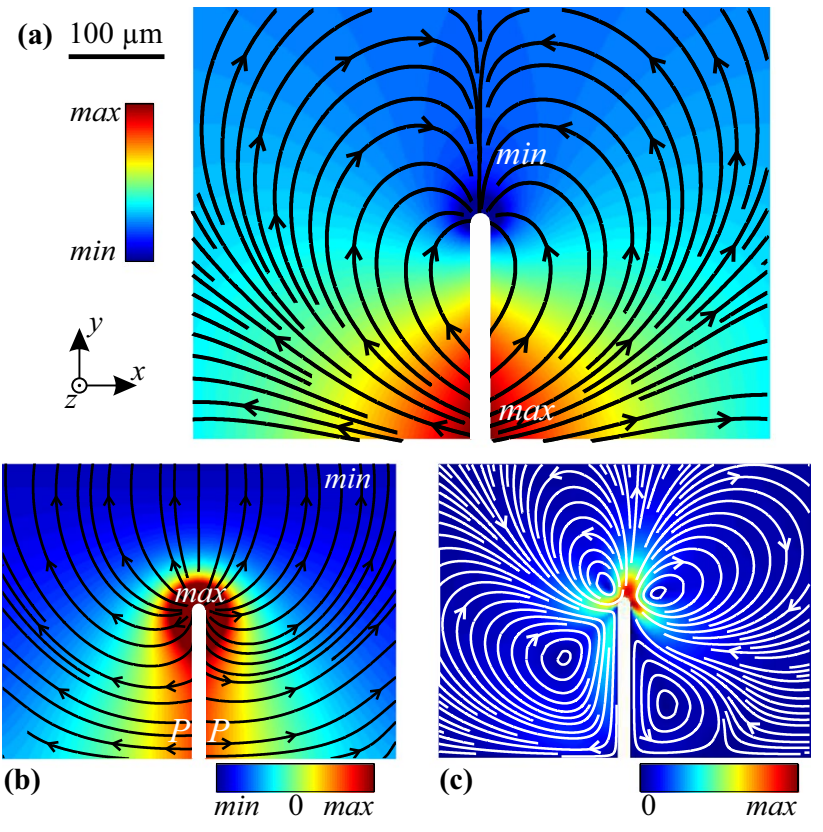

Fig. 8 a Color plot of the Gor'kov potential $U$ with vector and streamline plot of the force field $\mathbf{F}$ for copolymer particles, following from the simulation of Fig. 7. The streamlines correspond well to the tracked particle paths in Fig. 2d. b Color plot of the Gor'kov potential $U$ with vector and streamline plot of the force field $\mathbf{F}$ for hollow particles as in the experiment of Fig. 4d. c Acoustic streaming: color plot of the Lagrangian velocity magnitude with streamlines, corresponding to Fig. 6

The parameters $f_{1}, f_{2}$ of the yeast cells in our experiment are not precisely known, yet a quantitative estimation can be calculated from density and compressibility values in literature (Gherardini et al. 2005). Qualitatively, the behavior of yeast cells is similar to copolymer particles as discussed above and as experimentally confirmed in Fig. 2. Copolymer particles exhibit well-defined material properties (see Table 1 ) with known parameters $f_{1}=0.76$ and $f_{2}=0.034$. With these parameters for copolymer, the Gor'kov potential $U$ and the force field $\mathbf{F}$ are plotted in Fig. 8a. The force field illustrates the pressure minimum and velocity maximum attraction as discussed before. The streamlines of the force field were also plotted for a comparison with the tracked particle paths in Fig. 2d. The qualitative match between the modeled streamlines and the tracked particle paths is believed to confirm our model assumptions in this case. As discussed in Sect. 3.1.1, also the numerical model shows a strong increase in the acoustic radiation force toward the tip of the edge. Notably, the effect as modeled here occurs in a wide frequency range and is not depending on a sharp resonance frequency as common acoustophoretic traps with standing waves.

Furthermore, Fig. $8 \mathrm{~b}$ shows the Gor'kov potential and force field which follow for the hollow particles with $f_{1}=0.602, f_{2}=-0.362$ (Leibacher et al. 2014). Because of $f_{2}<0$, the model predicts a strong particle repulsion from the tip of the sharp edge, as clearly experienced in the experiments. However, the acoustic radiation force model cannot explain the experimentally observed attraction of some hollow particles to the two points on the left and right side at the base of the edge (marked with a $P$ ). This discrepancy might result from superposed acoustic streaming effects (as discussed in the next paragraph), near-field effects from the corner geometry, or effects which were missed by the simplification of the model.

Regarding acoustic streaming, numerical modeling approaches have been presented in literature (Möller 2013; Muller et al. 2012). Recently, studies on the acoustic streaming around oscillating sharp edge geometries have been published for frequencies of $461 \mathrm{~Hz}$ (Ovchinnikov et al. 2014) and $4.75 \mathrm{kHz}$ (Nama et al. 2014), also in combination with acoustic radiation forces. The results of these studies show that the complex streaming pattern depends on boundary conditions (Nama et al. 2014) as the geometry, the direction of vibration of the sharp edge, and the radius of curvature at the tip of the sharp edge, while the driving mechanism of the streaming is the rushing of the fluid from one side of the sharp edge to the other (Ovchinnikov et al. 2014). We implemented an analog numerical approach in Comsol Multiphysics to obtain an exemplary streaming pattern in Fig. 8c, which shows the acoustic streaming (Lagrangian velocity) for a sharp edge oscillation with amplitude $A$ in $y$-direction and amplitude $0.2 A$ in $x$-direction, so the shape and sense of rotation of the two strong upper vortices resemble the experimental results of Fig. 6. The observed asymmetry of the vortices in the model and experiment might be attributed to an edge vibration in both $x$ - and $y$-direction. The sensitive dependence on various boundary conditions might explain why some sharp edges showed experimentally a dominating radiation force and others a dominating streaming force, depending on the position-dependent vibration field magnitude and direction on the chip, and depending on manufacturing variations of the sharp edge. Further modeling is necessary for a full understanding and control of the phenomenon and the balance between acoustic radiation and streaming forces.

Furthermore, a transition from radiation-dominated to streaming-dominated particle kinetics has been reported (Barnkob et al. 2012) depending on the particle size. This transition determines whether the shown acoustophoretic trapping works also on particles smaller than yeast cells, e.g., bacteria and viruses.

Gor'kov's theory is actually only valid in an infinite acoustic domain far away from walls and other structures, which influence the acoustic radiation force (Wang and Dual 2012). This condition must be considered in the modeled situation, since the particles are close to a sharp edge. Gor'kov theory does not account for the reflections 


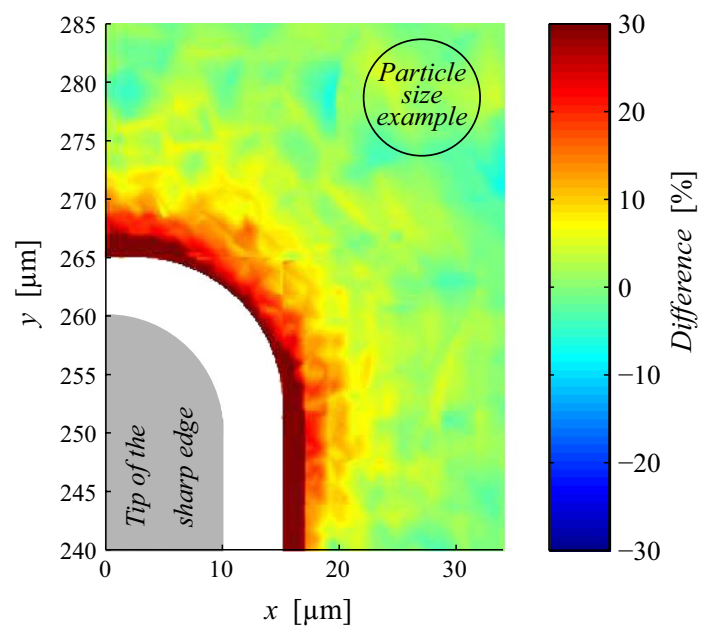

Fig. 9 Evaluation of Gor'kov's theory regarding wall effects on the tip of the sharp edge: The difference between the acoustic radiation force calculation with Eq. 5 and Gor'kov's approximation (Eq. 1) is mostly visible for particles which are very close to the sharp edge, where Eq. 5 predicts a higher attractive force than Gor'kov's model

of the scattered waves from the particle on the sharp edge, which might influence the acoustic radiation force, similar as two particles interact with each other by secondary acoustic forces (e. g. Bjerknes forces) (Laurell et al. 2007; Silva et al. 2014). Therefore, a validation of the Gor'kov approximation by the following more fundamental equation is reasonable. Neglecting fluid viscosity, the timeaveraged acoustic radiation force vector $\mathbf{F}=\left(F_{x}, F_{y}, F_{z}\right)$ can be calculated for a compressible particle of any shape as derived by Yosioka and Kawasima (Bruus 2012; Yosioka and Kawasima 1955):

$$
\begin{aligned}
\mathbf{F} & =-\int_{S}\left[\left(\frac{1}{2 \rho_{0} c_{0}^{2}}\left\langle p_{1}^{2}\right\rangle-\frac{1}{2} \rho_{0}\left\langle\mathbf{v}_{1} \cdot \mathbf{v}_{1}\right\rangle\right) \mathbf{n}\right. \\
& \left.+\rho_{0}\left\langle\left(\mathbf{n} \cdot \mathbf{v}_{1}\right) \mathbf{v}_{1}\right\rangle\right] \mathrm{d} S
\end{aligned}
$$

where the integration takes place over an arbitrary fixed surface $S$ enclosing the particle and the surface normal unit vector $\mathbf{n}$. The numerical accuracy and applicability of this approach are described in literature (Glynne-Jones et al. 2013). When the backscattering effects of walls are incorporated in $p_{1}$ and $\mathbf{v}_{1}$, the above equation also includes the influence of these effects on the acoustic radiation force. Fig. 9 shows a comparison between this equation and Gor'kov's approximation at an excitation frequency of 900 kHz. Whereas Gor'kov's approximation allows the convenient calculation of the force field directly from an acoustic background field (without simulated particle), the evaluation of Eq. 5 is more complex: For every $(x, y)$ point in the plot, a solid glass particle of radius $r=5 \mu \mathrm{m}$ was placed at $(x, y)$ within the 3D acoustic domain and the above equation was evaluated on the resulting field at the particle surface. The material glass was chosen here since it results in both a high $f_{1}$ and $f_{2}$, see Table 1 . The numerical mesh size was down to $1.5 \mu \mathrm{m}$ near the particle surface.

Figure 9 signifies that Gor'kov's theory is a good model with negligible error for particles which are $\sim 10 \mu \mathrm{m}$ or more away from the sharp edge, yet for closer particles, additional attractive forces come into play. Furthermore, it has to be noted that the above discussion was only an inviscid approximation. When the particle approaches the sharp edge as close as the acoustic (Stokes) boundary layer thickness $\delta \approx 0.6 \mu \mathrm{m}$ (Muller et al. 2012; Nama et al. 2014) (at $900 \mathrm{kHz}$ in water), additional viscous effects arise which are not considered in the above model.

\section{Conclusion}

Acoustofluidic cell and particle trapping on oscillating sharp edges in microfluidic domains have been observed. An oscillating sharp edge generates an acoustic field around its tip which is suitable to attract suspended particles by acoustic radiation forces. The acoustic radiation forces were described in experiments and numerical models, together with the earlier reported acoustic streaming on sharp edges.

Unlike common acoustofluidic traps, the method presented here offers geometric freedom with respect to the trapping location, which was found to be on the tip of a freely placeable sharp edge. Apart from the sharp edge structure, no further modifications are necessary right within the fluidic domain, which allows various designs of the microfluidic cavity. Since the effect is not based on a fluid resonance with an ultrasonic standing wave (as common acoustofluidic traps), reflecting channel walls and precise fluidic resonance frequency tuning are not required. The transducer can also be placed freely on any location on the chip.

Compared to other methods like DEP, magnetic, or optical tweezers, the presented approach is relatively simple to implement in a device and can be miniaturized to fit the lab-on-a-chip concept.

A numerical simulation of acoustic radiation forces was found to match qualitatively with the experiments. Further numerical work aims at a more precise modeling of the complex interplay between radiation and streaming forces. A model including the mechanics of silicon and the piezoelectric transducer would further resolve the time-harmonic motion of the sharp edge. This might explain whether the acoustic radiation or streaming forces dominate on a specific sharp edge, depending on the mode of vibration, geometry, and material properties. Further numerical work might also address the influence of clusters of agglomerated particles (as in Fig. 2c) on the acoustic field and radiation forces. 
Future work can address the acoustofluidics and cell trapping around free-standing posts or tips (rather than sharp edges). Acoustic tweezers might be realized with a moveable tip, whose particle attraction can be turned on/ off by ultrasonic excitation for pick and place operations of particles. From a biological perspective, miniaturization for trapping of single cells is interesting as well as applied studies with cell trapping and perfusion.

Furthermore, future experimental work can approach particle separations by edge attraction/repulsion (depending on the particle properties) and a more defined excitation of the sharp edges, since the large bulk piezoelectric transducer as shown here resulted in a nonuniform and not well-defined vibration field on the chip.

Finally, the acoustofluidic phenomena of acoustic radiation and streaming forces around oscillating sharp edges are believed to offer promising capabilities for cell and particle handling at the microscale.

\section{References}

Ferroperm piezoceramics a/s, www.ferroperm-piezo.com

Ahmed D, Mao X, Shi J, Juluri BK, Huang TJ (2009) A millisecond micromixer via single-bubble-based acoustic streaming. Lab Chip 9:2738-2741

Augustsson P, Barnkob R, Wereley ST, Bruus H, Laurell T (2011) Automated and temperature-controlled micro-piv measurements enabling long-term-stable microchannel acoustophoresis characterization. Lab Chip 11:4152-4164

Barnkob R, Augustsson P, Laurell T, Bruus H (2010) Measuring the local pressure amplitude in microchannel acoustophoresis. Lab Chip 10:563-570

Barnkob R, Augustsson P, Laurell T, Bruus H (2012) Acoustic radiation-and streaming-induced microparticle velocities determined by microparticle image velocimetry in an ultrasound symmetry plane. Phys Rev E 86(5):056,307

Bruus H (2012) Acoustofluidics 2: perturbation theory and ultrasound resonance modes. Lab Chip 12:20-28

Bruus H (2012) Acoustofluidics 7: the acoustic radiation force on small particles. Lab Chip 12:1014-1021

Christakou AE, Ohlin M, Vanherberghen B, Khorshidi MA, Kadri N, Frisk T, Wiklund M, Önfelt B (2013) Live cell imaging in a micro-array of acoustic traps facilitates quantification of natural killer cell heterogeneity. Integr Biol 5(4):712-719

Dual J, Hahn P, Leibacher I, Moller D, Schwarz T (2012) Acoustofluidics 6: experimental characterization of ultrasonic particle manipulation devices. Lab Chip 12:852-862

Dual J, Möller D (2012) Acoustofluidics 4: piezoelectricity and application in the excitation of acoustic fields for ultrasonic particle manipulation. Lab Chip 12(3):506-514

Evander M, Johansson L, Lilliehorn T, Piskur J, Lindvall M, Johansson S, Almqvist M, Laurell T, Nilsson J (2007) Noninvasive acoustic cell trapping in a microfluidic perfusion system for online bioassays. Anal Chem 79(7):2984-2991

Evander M, Nilsson J (2012) Acoustofluidics 20: applications in acoustic trapping. Lab Chip 12:4667-4676

Gherardini L, Cousins CM, Hawkes JJ, Spengler J, Radel S, Lawler H, Devcic-Kuhar B, Gröschl M, Coakley WT, McLoughlin AJ (2005) A new immobilisation method to arrange particles in a gel matrix by ultrasound standing waves. Ultrasound Med Biol 31(2):261-272

Glynne-Jones P, Mishra PP, Boltryk RJ, Hill M (2013) Efficient finite element modeling of radiation forces on elastic particles of arbitrary size and geometry. J Acoust Soc Am 133(4):1885-1893

Gor'kov LP (1962) On the forces acting on a small particle in an acoustical field in an ideal fluid. Sov Phys Dokl 6(9):773-775

Gralinski I, Raymond S, Alan T, Neild A (2014) Continuous flow ultrasonic particle trapping in a glass capillary. J Appl Phys 115(5):054,505

Grier DG (2003) A revolution in optical manipulation. Nature 424(6950):810-816

Haeberle S, Mark D, von Stetten F, Zengerle R (2012) Microfluidic platforms for lab-on-a-chip applications. In: Microsystems and Nanotechnology. Springer Berlin Heidelberg, pp 853-895

Hammarström B, Evander M, Barbeau H, Bruzelius M, Larsson J, Laurell T, Nilsson J (2010) Non-contact acoustic cell trapping in disposable glass capillaries. Lab Chip 10(17):2251-2257

Hashmi A, Yu G, Reilly-Collette M, Heiman G, Xu J (2012) Oscillating bubbles: a versatile tool for lab on a chip applications. Lab Chip 12(21):4216-4227

Hu J, Ong L, Yeo C, Liu Y (2007) Trapping, transportation and separation of small particles by an acoustic needle. Sens Actuators A Phys 138(1):187-193

Hu J, Yang J, Xu J (2004) Ultrasonic trapping of small particles by sharp edges vibrating in a flexural mode. Appl Phys Lett 85(24):6042-6044

Huang PH, Nama N, Mao Z, Li P, Rufo J, Chen Y, Xie Y, Wei CH, Wang L, Huang TJ (2014) A reliable and programmable acoustofluidic pump powered by oscillating sharp-edge structures. Lab Chip 14(22):4319-4323

Huang PH, Xie Y, Ahmed D, Rufo J, Nama N, Chen Y, Chan CY, Huang TJ (2013) An acoustofluidic micromixer based on oscillating sidewall sharp-edges. Lab Chip 13:3847-3852

Johann RM (2006) Cell trapping in microfluidic chips. Anal Bioanal Chem 385(3):408-412

Karimi A, Yazdi S, Ardekani A (2013) Hydrodynamic mechanisms of cell and particle trapping in microfluidics. Biomicrofluidics 7(2):021-501

Laurell T, Petersson F, Nilsson A (2007) Chip integrated strategies for acoustic separation and manipulation of cells and particles. Chem Soc Rev 36:492-506

Leibacher I, Dietze W, Hahn P, Wang J, Schmitt S, Dual J (2014) Acoustophoresis of hollow and core-shell particles in two-dimensional resonance modes. Microfluid Nanofluid 16(3):513-524

Leibacher I, Dual J (2014) Acoustofluidic micro devices for cell and particle trapping. In: proceedings of the 40th international conference on micro and nano engineering (MNE), 22-26 Sept 2014, Lausanne, p. 191

Leibacher I, Dual J (2014) Cell trapping on acoustofluidic edge structures. In: proceedings of the acoustofluidics 2014 meeting, 11-12 Sept 2014, Prato

Leibacher I, Schatzer S, Dual J (2014) Impedance matched channel walls in acoustofluidic systems. Lab Chip 14:463-470

Lenshof A, Evander M, Laurell T, Nilsson J (2012) Acoustofluidics 5: building microfluidic acoustic resonators. Lab Chip 12:684-695

Li S, Glynne-Jones P, Andriotis OG, Ching KY, Jonnalagadda US, Oreffo RO, Hill M, Tare RS (2014) Application of an acoustofluidic perfusion bioreactor for cartilage tissue engineering. Lab Chip 14(23):4475-4485

Lieu VH, House TA, Schwartz DT (2012) Hydrodynamic tweezers: impact of design geometry on flow and microparticle trapping. Anal Chem 84(4):1963-1968

Lighthill J (1978) Acoustic streaming. J sound vib 61(3):391-418

Liu Y, Hu J (2009) Ultrasonic trapping of small particles by a vibrating rod. IEEE Trans Ultrason Ferroelectr Freq Control 56(4):798-805 
Manneberg O, Vanherberghen B, Svennebring J, Hertz HM, Önfelt B, Wiklund M (2008) A three-dimensional ultrasonic cage for characterization of individual cells. Appl Phys Lett 93(6):063901

Marin A, Rossi M, Rallabandi B, Wang C, Hilgenfeldt S, Kähler CJ (2015) Three-dimensional phenomena in microbubble acoustic streaming. Phys Rev Appl 3(4):0410,01

Marmottant P, Hilgenfeldt S (2004) A bubble-driven microfluidic transport element for bioengineering. Proc Natl Acad Sci U S A 101(26):9523-9527

McSkimin HJ, Andreatch P Jr (1964) Elastic moduli of silicon vs hydrostatic pressure at $25.0^{\circ} c$ and $-195.8^{\circ} c$. J Appl Phys 35(7):2161-2165

Möller DB (2013) Acoustically driven particle transport in fluid chambers. Ph.D. thesis, Eidgenössische Technische Hochschule ETH Zürich, Nr. 21644

Muller PB, Barnkob R, Jensen MJH, Bruus H (2012) A numerical study of microparticle acoustophoresis driven by acoustic radiation forces and streaming-induced drag forces. Lab Chip 12(22):4617-4627

Nama N, Huang PH, Huang TJ, Costanzo F (2014) Investigation of acoustic streaming patterns around oscillating sharp edges. Lab Chip 14(15):2824-2836

Nilsson J, Evander M, Hammarström B, Laurell T (2009) Review of cell and particle trapping in microfluidic systems. Anal Chim Acta 649(2):141-157

Oberti S, Neild A, Dual J (2007) Manipulation of micrometer sized particles within a micromachined fluidic device to form twodimensional patterns using ultrasound. J Acoust Soc Am 121(2):778-785

Oberti S, Neild A, Ng TW (2009) Microfluidic mixing under low frequency vibration. Lab Chip 9(10):1435-1438

Ovchinnikov M, Zhou J, Yalamanchili S (2014) Acoustic streaming of a sharp edge. J Acoust Soc Am 136(1):22-29
Rogers P, Neild A (2011) Selective particle trapping using an oscillating microbubble. Lab Chip 11:3710-3715

Sadhal S (2012) Acoustofluidics 13: analysis of acoustic streaming by perturbation methods. Lab Chip 12(13):2292-2300

Sadhal S (2012) Acoustofluidics 16: acoustics streaming near liquidgas interfaces: drops and bubbles. Lab Chip 12(16):2771-2781

Salieb-Beugelaar GB, Simone G, Arora A, Philippi A, Manz A (2010) Latest developments in microfluidic cell biology and analysis systems. Anal Chem 82(12):4848-4864

Silva, Glauber T, Henrik Bruus (2014) Acoustic interaction forces between small particles in an ideal fluid. Physical Review E 90.6:063007

Vanherberghen B, Manneberg O, Christakou A, Frisk T, Ohlin M, Hertz HM, Onfelt B, Wiklund M (2010) Ultrasound-controlled cell aggregation in a multi-well chip. Lab Chip 10:2727-2732

Voldman J (2006) Electrical forces for microscale cell manipulation. Annu Rev Biomed Eng 8:425-454

Wang J, Dual J (2012) Theoretical and numerical calculation of the acoustic radiation force acting on a circular rigid cylinder near a flat wall in a standing wave excitation in an ideal fluid. Ultrasonics 52(2):325-332

West J, Becker M, Tombrink S, Manz A (2008) Micro total analysis systems: latest achievements. Anal Chem 80(12):4403-4419

Whitesides GM (2006) The origins and the future of microfluidics. Nature 442(7101):368-373

Wiklund M (2012) Acoustofluidics 12: biocompatibility and cell viability in microfluidic acoustic resonators. Lab Chip 12:2018-2028

Wiklund M, Green R, Ohlin M (2012) Acoustofluidics 14: applications of acoustic streaming in microfluidic devices. Lab Chip 12(14):2438-2451

Yosioka K, Kawasima Y (1955) Acoustic radiation pressure on a compressible sphere. Acustica 5:167-173 